\title{
Control biológico de la Antracnosis causada por Colletotrichum gloeosporioides (Penz. y Sacc.) en Tomate de Árbol (Solanum betaceum Cav.) mediante hongos endófitos antagonistas.
}

\author{
Biological control of Anthracnose Colletotrichum gloeosporioides (Penz y Sacc) on Tree Tomato \\ (Solanum betaceum Cav.) by antagonist endophyte fungi.

\section{Ernesto Delgado Fernández*, Santiago Vásquez Matute} \\ Centro de Investigación en Valoración de la Biodiversidad CIVABI, Universidad Politécnica Salesiana, Paute, Ecuador. PBX: (593) 72854972 \\ * Autor para correspondencia: mdelgado@ups.edu.ec
}

Artículo recibido el 15 de mayo de 2010. Aceptado tras revisión el 20 de junio de 2010 .

\begin{abstract}
Resumen
La antracnosis, en el tomate de árbol (Solanum betaceum) conocida por nuestros agricultores como "ojo de pollo" se produce por un hongo del género Colletotrichum Sp. es una enfermedad que causa pérdidas incalculables, y hasta el momento su control, se hace mediante productos químicos, que en algunos casos son nocivos y atentan contra el medio ambiente. El uso de microorganismos como inhibidores del desarrollo de microorganismos fitopatógenos, se presenta como una alternativa para el control del patógeno. En esta investigación se emplearon tres hongos endófitos antagonistas aislados de dos plantas consideradas medicinales: la Chuquiragua (chuquiragua jussieui) y Ñachag (Bidens andícola Kunth) se aplican tres tratamientos con hongos endófitos, el primero se trabaja con el género Nigrospora. Sp, el segundo con una cepa del mismo género pero diferente especie, mientras que el tercero es un hongo endófito, considerado en su taxonomía como micelio estéril.Además se empleó un testigo referencial (inóculo del hongo Colletotrichum) y un testigo absoluto (sin inóculo). El bioensayo se realiza en condiciones semicontroladas de temperatura y humedad relativa. El análisis estadístico determina que el mejor tratamiento es el segundo, seguido del primer tratamiento; el tercer tratamiento y el testigo absoluto se presentan estadísticamente similares, las diferencias significativas se dan entre el tratamiento dos y el testigo referencial. Estos resultados evidencian la disminución y control de los daños causados en Solanum betaceum, se puede afirmar que dicha cepa no interfiere negativamente en la fisiología del cultivo, y posiblemente promueva un mejor desarrollo de la biomasa, ya que las plantas pertenecientes a este tratamiento se observaron con mayor vigor. Se recomienda probar inóculos combinados de hongos endófitos antagonistas, evaluar el potencial antagónico, a campo abierto y en condiciones ambientales diversas, además de evaluar el uso de macro y micro nutrientes en la potenciación de la efectividad del biocontrol.
\end{abstract}

Palabras clave: Antracnosis, endofitismo, antagonismo

\begin{abstract}
Anthracnose on tomato tree (Solanum betaceum) is known by our farmers as "chicken eye". It is produced by fungi of the genus Colletotrichum Sp. It is a disease causing major losses. Up to now it is controlled by the use of chemical products, which are sometimes harmful for people and the environment. The use of microorganisms for inhibiting the development of phytopathogenic microorganisms is an alternative control. Three isolated antagonist endophyte fungi isolated on two medicinal plants were used in this research: Chuquiragua (chuquiragua jussieui) and Ñachag (Bidens andícola Kunth). Three treatments with endophyte fungi were applied: the first treatment involves the genus Nigrospora. Sp; the second involves the second stump of Nigrospora Sp; and the third is an endophyte fungus taxonomically considered as a sterile mycelium. Also, a referential witness was used (inoculum of Colletotrichum) and an absolute witness (without inoculum). The bioassay is conducted in semi-controlled conditions of relative temperature and humidity. Statistical analysis finds that the best treatment is the second, followed by the first. The third treatment and the absolute witness are statistically similar while significant differences are found in the second treatment and the referential witness. These results are evidence of decrease and control of damaged caused by C. Gloesporoides on Solanum betaceum. It can be stated that such stump does not influence the physiology of the crop negatively and may promote an improved development of the biomass inasmuch as plants under treatment are observed to be healthier. It is recommended to test a combination of inoculums of antagonist endophyte fungi, to evaluate their antagonistic potential in open spaces and different environmental conditions, as well as to evaluate the use of macro and micro nutrients for a more effective biocontrol.
\end{abstract}

Keywords: Anthracnose, endophyte, antagonistic

Forma sugerida de citar: Delgado Fernández y Vásquez Matute. 2010, Control biológico de la Antracnosis causada por
Colletotrichum gloeosporioides (Penz. y Sacc.) en Tomate de Árbol (Solanum betaceum Cav.)
mediante hongos endófitos antagonistas. La Granja II (I). Pp. 36-43 


\section{Introducción}

Hace algunos años se presentó un problema fitopatológico muy grave en la zona del austro ecuatoriano, la producción de tomate de árbol (Solanum betaceum) fue afectada, por una enfermedad conocida por los agricultores como ojo de pollo, que ocasionó la destrucción de grandes extensiones de cultivo, esta enfermedad es causada por un hongo "Colletotrichum" aunque en alguna etapa "Colletotrichum acutatum", la sintomatología, se presenta con agresividad, en toda la planta, aunque es más evidente en el fruto, en esta investigación se caracterizó al patógeno incluso en las flores. La identificación, del patógeno se basa en sus características morfológicas, especialmente características conidiales, presencia de setas, esclerocios y forma de los apresorios (Bailey y Jeger, 1992). Es posible mejorar su estudio taxonómico mediante el uso de pruebas moleculares y ADN ribosomal. El cultivo de tomate de árbol (Solanum betaceum) en la actualidad, se maneja y controla, mediante el uso de productos químicos que en algunos casos, resultan ser altamente tóxicos, ya que contribuyen al deterioro del medio ambiente, y consecuentemente a la mala calidad del fruto, el uso de microorganismos como agentes de control ha despertado mucho interés para la fitopatología moderna, esta investigación tiene su sustento en el uso de hongos endófitos como agentes de control de la antracnosis Colletotrichum. Con este propósito, se parte de la selección de estos microorganismos con potencial antagónico, y mediante ensayos in vitro, se determinan los porcentajes de inhibición, y su capacidad antagónica (biocontroladores), los hongos antagonistas en este caso fueron aislados como hongos endofitos de dos plantas medicinales Chuquiragua Jussieui y Bidens Andícola Kunth, plantas endémicas del parque nacional "El Cajas" en Cuenca, Ecuador.

Estudios acerca de la actividad biológica de estos microorganismos demuestran que algunas cepas, particularmente de la taxa "Mycelia sterilia" (micelios estériles), manifiestan una elevada actividad antagónica in-vitro frente a Colletotrichum (Delgado, 2009).

El uso de estos aislados promisorios para el biocontrol de la antracnosis en tomate de árbol Sola- num betaceum Cav. en condiciones semicontroladas de temperatura y humedad relativa, es el propósito de esta investigación.

\section{Materiales y Métodos}

\section{I Ubicación del lugar de ensayo}

Esta investigación, se llevó a cabo en los Laboratorio del Centro de Investigación y Valoración de la Biodiversidad CIVABI del Campus Juan Lunardi de Paute, Provincia del Azuay en Ecuador; se utilizó además un espacio adecuado, y destinado a la creación de un microclima, que se establece bajo las siguientes características: temperatura promedio $20^{\circ} \mathrm{C}$, y humedad relativa $70 \%$

Asimismo, las muestras fueron tomadas en Paute, que se halla a una altitud de $2230 \mathrm{msnm}$; con una temperatura que oscila entre los $16-22^{\circ} \mathrm{C}$; una humedad relativa: $60 \%$ y $700 \mathrm{~mm}$ anuales en precipitación.

\subsection{Material Biológico}

Se utilizaron tres cepas de hongos endofitos antagonistas, y una cepa patogénica de Colletotrichum gloeosporioides que se aisló de frutos y hojas enfermas de tomate de árbol (Solanum betaceum Cav.) con síntomas de la enfermedad.

Se experimentó con material vegetal de (Solanum betaceum Cav.), variedad Amarillo puntón, de tres meses de edad, estas plantas fueron sometidas a un proceso de desinfección con $\mathrm{CuSO}_{4} \cdot 5 \mathrm{H}_{2} \mathrm{O}$, posteriormente se trasplantó a fundas plásticas que contienen sustrato, con características de un terreno franco arenoso, el mismo que se desinfectó utilizando Oxicarboxin + PCNB. El material vegetal se ubicó en el área destinada al ensayo, bajo condiciones semi controladas de temperatura y humedad relativa, las plantas pasaron por un proceso de climatización de un mes. Como posibles agentes de control de la antracnosis se emplearon aislados promisorios codificados como $\mathrm{Al} 3$ y A20, correspondientes al género Nigrospora Sp.y HXI micelio estéril, las cepas, pasaron por un proceso de análisis de actividad biológica riguroso, en donde se 
pudo evidenciar su capacidad antagónica in vitro en el proceso de investigación (Delgado, 2009).

Se inocularon los hongos endófitos antagonistas y el patógeno, mediante aspersión de suspensiones de esporas.

\subsection{Preparación desuspensiones monospóricas.}

Para la obtención de las suspensiones se parte de cultivos monospóricos con períodos de quince días de cultivo, (los primeros cinco días en completa obscuridad, y luego hasta el día quince, con un fotoperiodo normal de dieciséis horas luz, con el objeto de estimular la esporulación), los cultivos se hicieron en matraces de $250 \mathrm{ml}$, con $40 \mathrm{ml}$ de PDA, los mismos se lavaron cinco veces consecutivas, con $100 \mathrm{ml}$ de agua destilada estéril cada uno, obteniéndose 500 $\mathrm{ml}$ de suspensión por cada matraz de $250 \mathrm{ml}$, posteriormente se filtraron con una gasa estéril (Mendoza, 2003). Se adicionó Tween 20 al 0,5\%, y se determinó la concentración de conidias o ufc (unidades formadoras de colonias) por ml, para el efecto se utilizó un hematocimetro o cámara de Neubauer. (Chávez, 2007; Cañedo y Ames, 2004), ver Figuras I y 2.

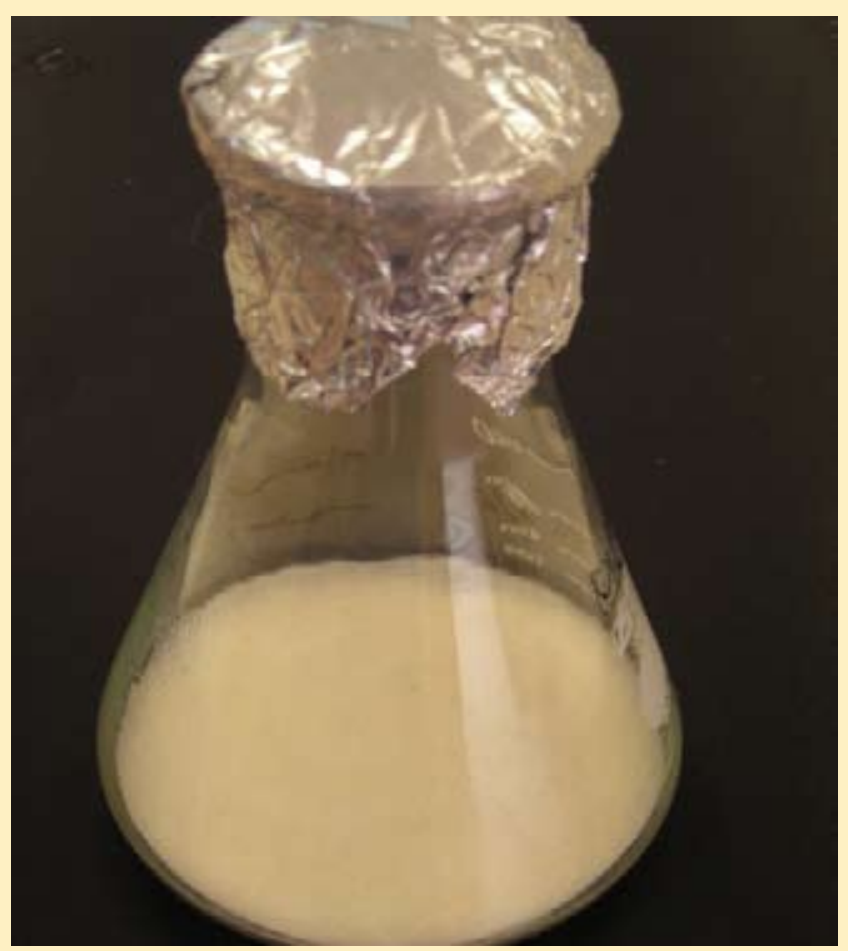

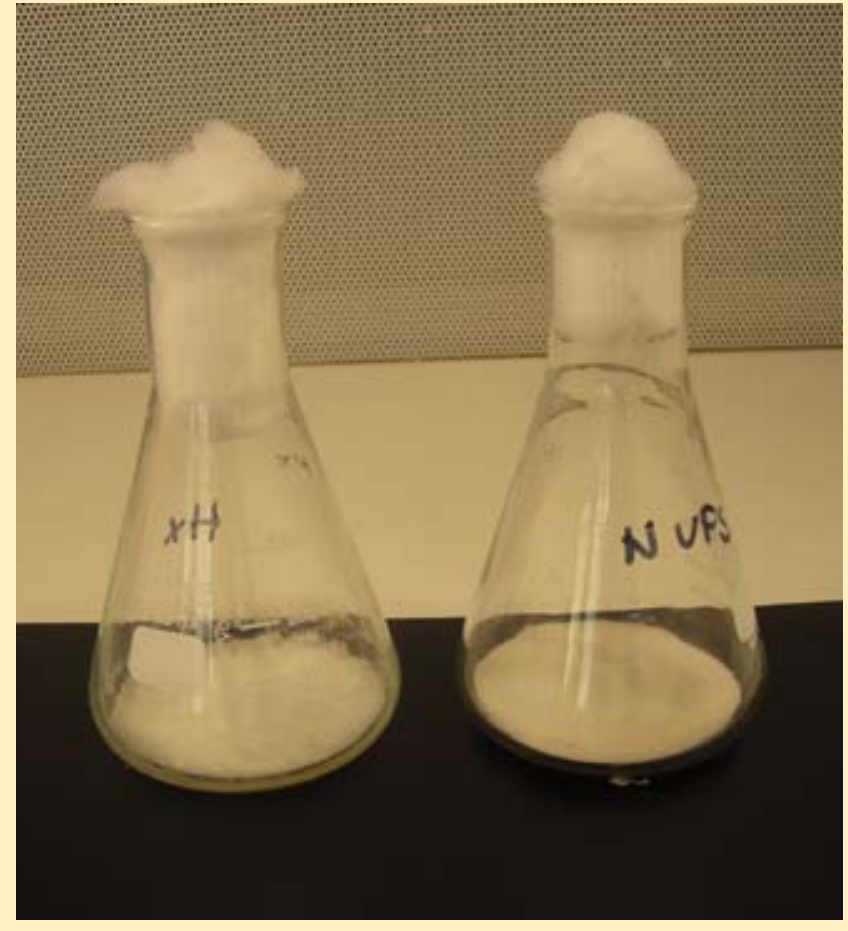

Figuras I y 2. Fotos de cultivos monospóricos de hongos endófitos antagonistas en matraces. Fuente: Ernesto Delgado Fernández.

\subsection{Tratamientos}

Se aplicaron tres tratamientos, un testigo referencial y un testigo absoluto, según se detalla en la Tabla I.

Tabla I.Tratamientos efectuados en la investigación.

\section{TRATAMIENTOS}

\begin{tabular}{ll}
\hline Tratamiento I & Suspensión de esporas de \\
& Nigrospora. Sp: cepa A13 \\
& (solución: I,7x $10^{3}$ ufc/ml)
\end{tabular}

Tratamiento 2 Suspensión de esporas de Nigrospora sp: cepa A20 (solución I,9x10 ufc/ml)

Tratamiento 3: $\quad$ Suspensión de esporas micelio estéril: cepa HXI (solución I, $0 \times 10^{3}$ ufc/ml)

Testigo Referencial (inoculó del patógeno) "Colletotrichum gloesporoides"

Testigo Absoluto (sin inóculo) 


\subsection{Aplicación de inóculos}

Se aplican suspensiones de $10 \mathrm{ml}$ del patógeno, "Colletotrichum gloeosporioides" en cada planta, dicha aplicación se hace en todos los tratamientos, excepto en el testigo absoluto, 24 horas después de la aplicación, se inoculó las suspensiones de esporas de los hongos endófitos antagonistas citados, se aplicaron $10 \mathrm{ml}$ de suspensión por cada planta, inoculando de esta forma la cepa $\mathrm{A} \mid 3$ en el primer tratamiento, $\mathrm{A} 20$ en el segundo tratamiento y $\mathrm{HXI}$ en el tercer tratamiento.

Las plantas se mantuvieron bajo condiciones semicontroladas, cuya temperatura promedio fué de $22^{\circ} \mathrm{C}$ y la humedad relativa (HR) tuvo un promedio de $76 \%$ el riego se realizó mediante aspersión con agua blanda, para favorecer la penetración del patógeno, y según las necesidades de las plantas y para mantener las condiciones de temperatura y humedad. La evaluación del proceso se hizo después de un mes; además se consideró, la altura de la planta, el peso de la biomasa fresca, la deficiencia en el desarrollo radicular y el número de hojas Infestadas.

\subsection{Diseño experimental y análisis estadístico}

Se utilizó un diseño experimental completamente al azar, se consideran 3 tratamientos, un testigo referencial y un testigo absoluto, se utilizaron cuatro plantas de Solanum betaceum por tratamiento. El análisis de los resultados se realizó mediante análisis de varianza, en los casos que estadísticamente hubo diferencias significativas, se compara los tratamientos, mediante la prueba de Tukey al $95 \%$, con asistencia del paquete estadístico MINITAB I5.

\subsection{Prueba de patogenicidad y taxonomía}

Se identificaron la morfología y las estructuras características del hongo Colletotrichum. La taxonomía del agente causal de la antracnosis, es confusa y se presentan cerca de 600 sinónimos de esta especie (Kriket et al., 2008). Una de las características del género, es la presencia acérvulos en forma de disco o almohadilla, cerosos, subepidermales, típicamente de color salmón, con setas en el borde del acérvulo y entre los conidióforos, conidias hialinas, unicelulares, ovoides u oblongas (Barnett y Huntter, 1998).

A continuación se describe la taxonomía del patógeno (Figuras 3 y 4): Reino: Fungi. Orden: Melanconiales. División: Ascomycota familia: Melanconiaceae género: Colletotrichum. Especie: gloeosporioides (Penz y Sacc).

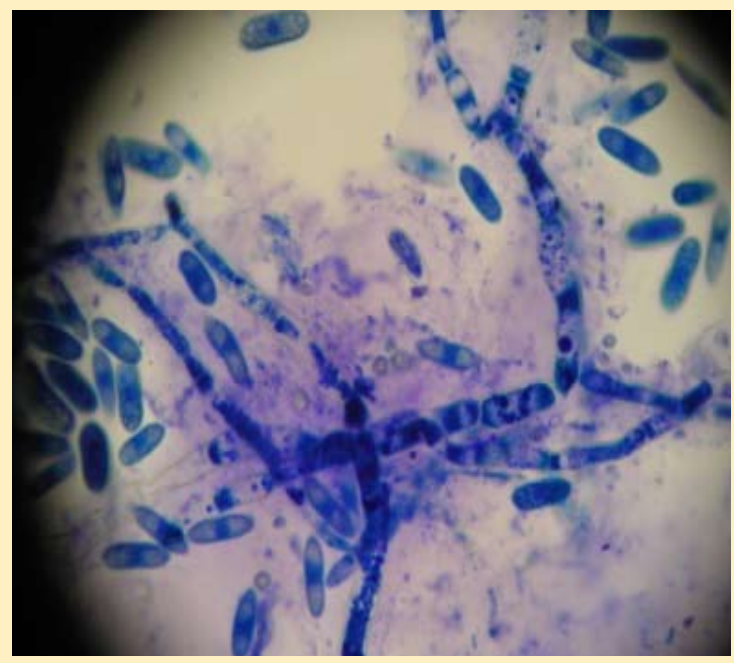

Figura 3. Foto al microscopio de micelios y conidios del hongo del género Colletotrichum. Fuente: Ernesto Delgado Fernández

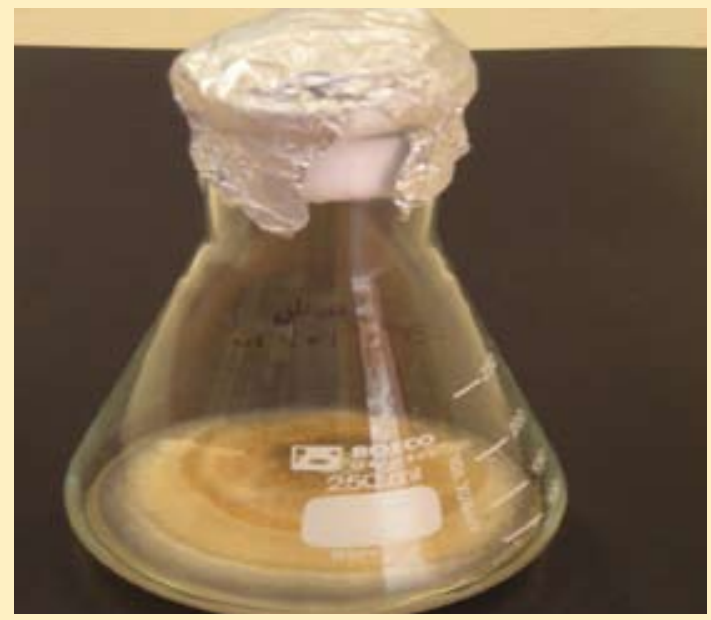

Figura 4. Hongo del género Colletotrichum aislado del fruto del tomate de árbol Solanum betaceum Cav. Fuente: Ernesto Delgado Fernández

\subsection{Características Fitopatológicas}

Las plantas inoculadas con el patógeno presentan cambios fisiológicos, marcados síntomas de marchitez, amarillamiento, áreas necróticas de color marrón en los bordes, y encapuchamiento. (Ver Fig. 5, 6, 7,8). 

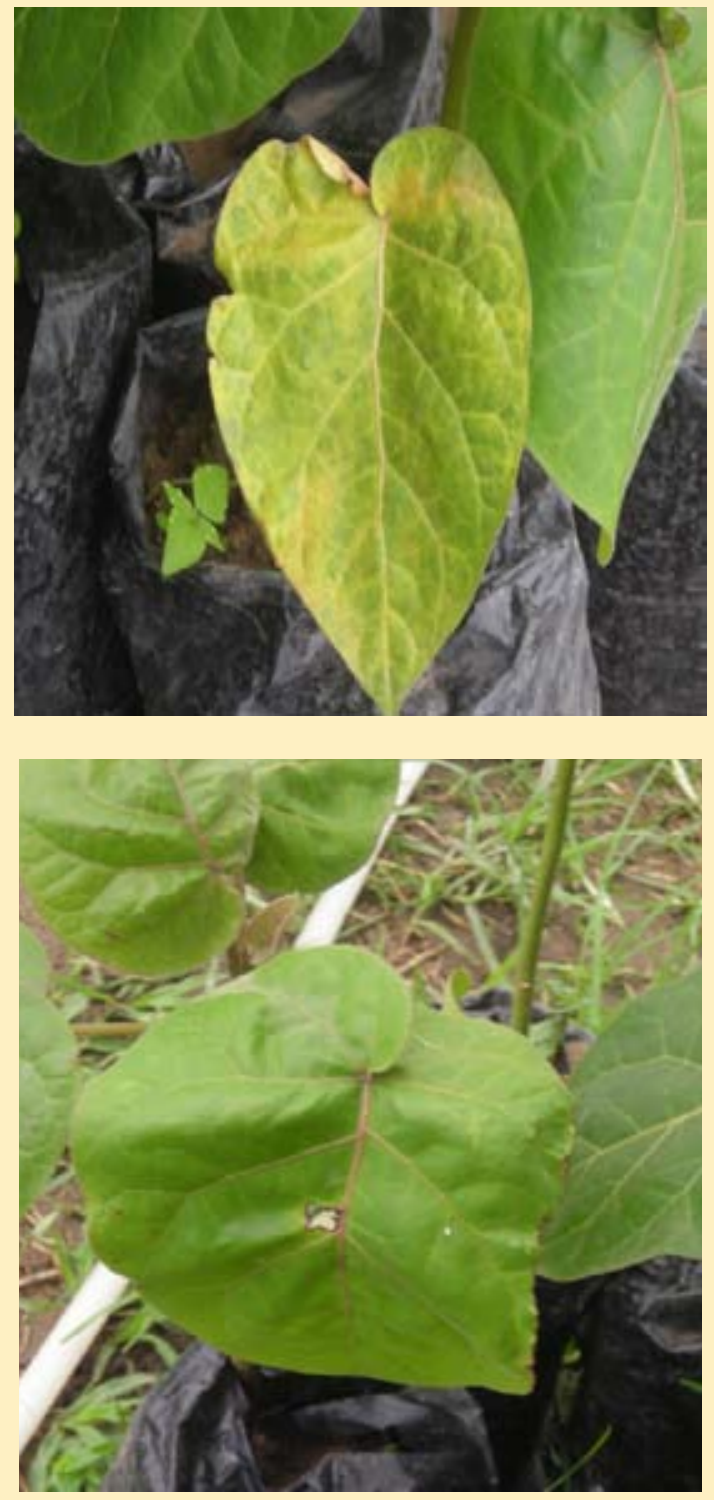

Figura 5 y 6 . Fotos de plantas de tomate Solanum betaceae con síntomas de antracnosis. Fuente: Ernesto Delgado Fernández

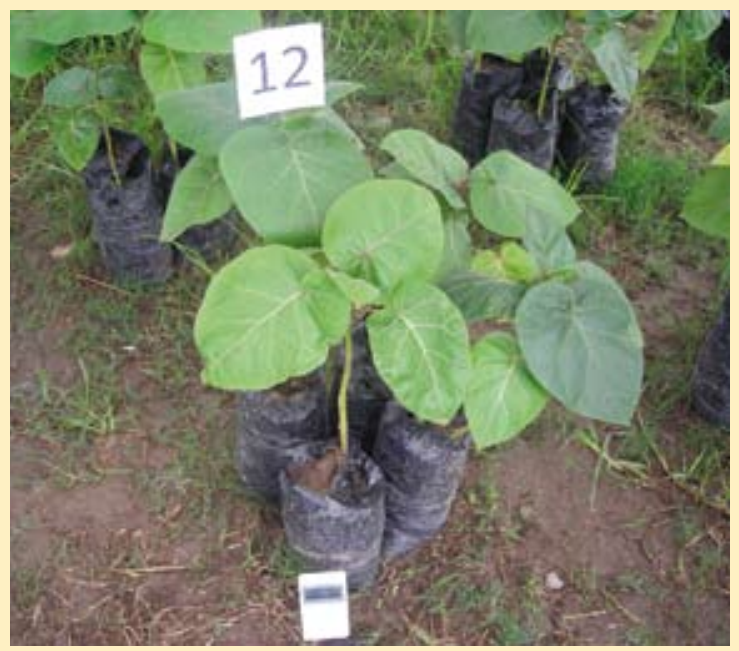

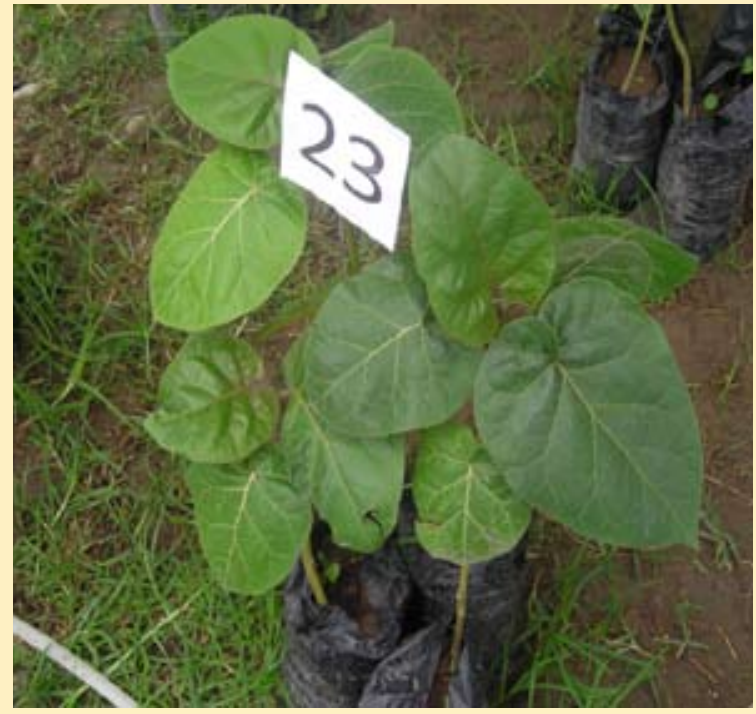

Figura 7 y 8 . Plantas de tomate Solanum Betaceae Cav. con inóculos de los testigos referencial y absoluto. Fuente: Ernesto Delgado Fernández

La Antracnosis Colletotrichum gloeosporioides (Penz.) en la presente investigación se evalúa fitopatologicamente, por los daños en hojas y ramas, ya que se utilizaron plantas de tres meses de edad, pero en plantaciones en producción, el daño es más notorio en los frutos (Figura 9), los cuales son afectados en todos sus estados (Saldarriaga, 1997) aunque, en el análisis micológico de flores se determinó la presencia del agente patógeno.

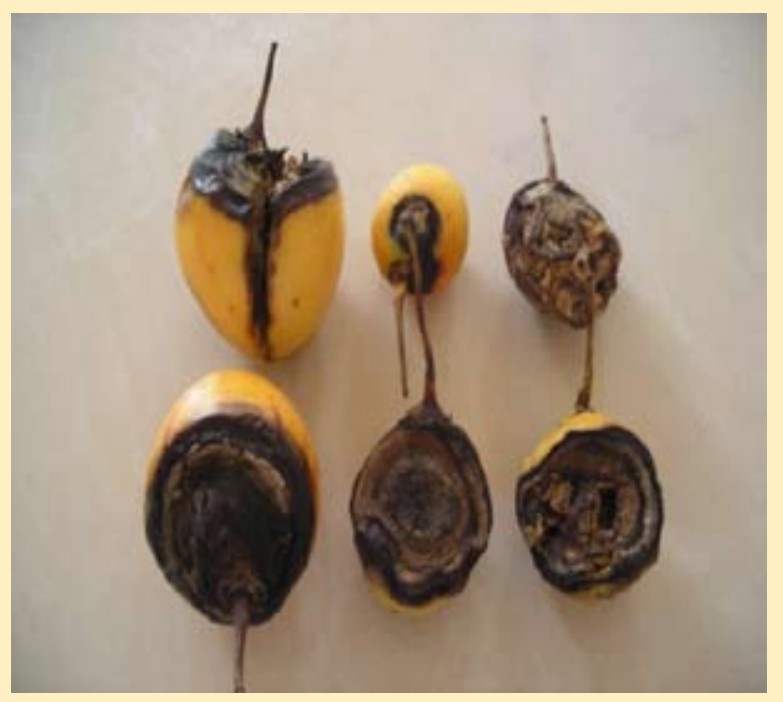

Figura 9. Frutos de tomate de árbol Solanum betaceae con síntomas de antracnosis. Fuente: Ernesto Delgado Fernández 


\section{Resultados}

\section{I Numero de hojas infestadas.}

La evaluación del grado de infestación se hizo mediante observación, de los caracteres fitopatológicos de la planta, se contó el número de hojas con síntomas de la enfermedad por cada tratamiento. Se obtuvo que por un análisis ANOVA ( $F=2,14 \quad P=$ 0,127 ) a los 30 días de iniciado el experimento, se revela que si existe estadísticamente diferencia significativa entre los tratamientos. El mejor comportamiento se presenta con el aislado A20 de Nigrospora Sp, que corresponde al "Tratamiento 2" difiere significativamente con el resto de tratamientos, según se evidencia en la (Figuras 10, II y 12) y de acuerdo a la prueba de Tukey al 95\%; el "Tratamiento I" igualmente, difiere estadísticamente, corresponde a la cepa A 3 de Nigrospora Sp. La diferencia significativa estadísticamente es mayor con el testigo referencial que corresponde a Colletotrichum.

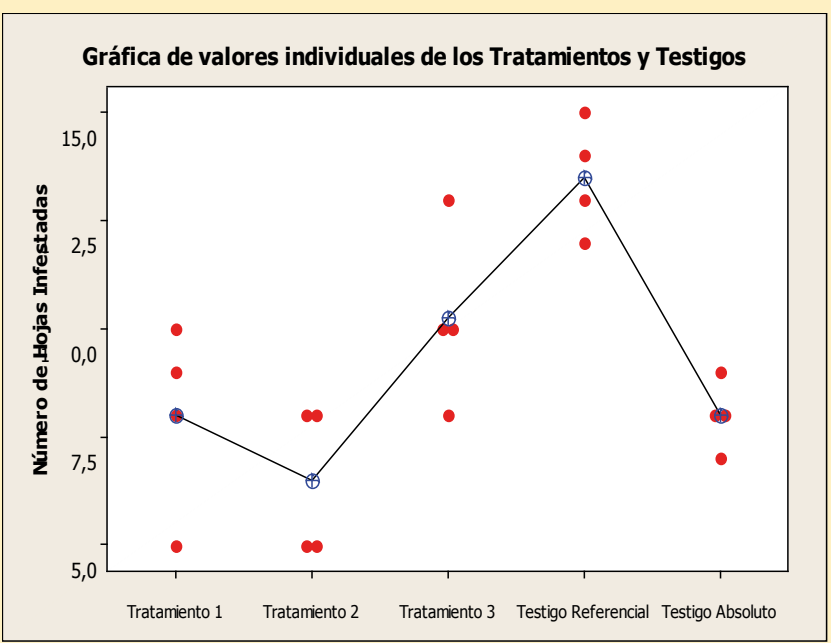

Figura I0.Valores individuales de los tratamientos y testigos. Fuente: Ernesto Delgado.

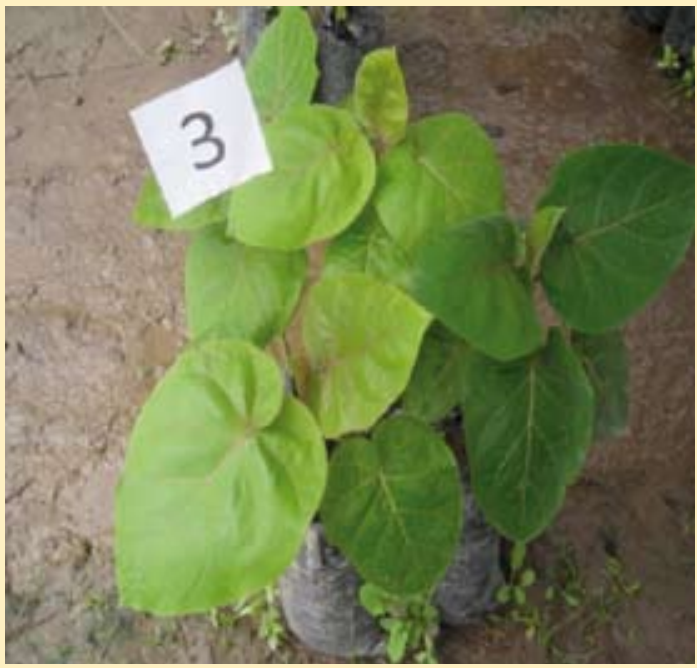

Figura II. Fotos de plantas Solanum betaceae, con inóculos de esporas Nigrospora. Fuente: Ernesto Delgado Fernández

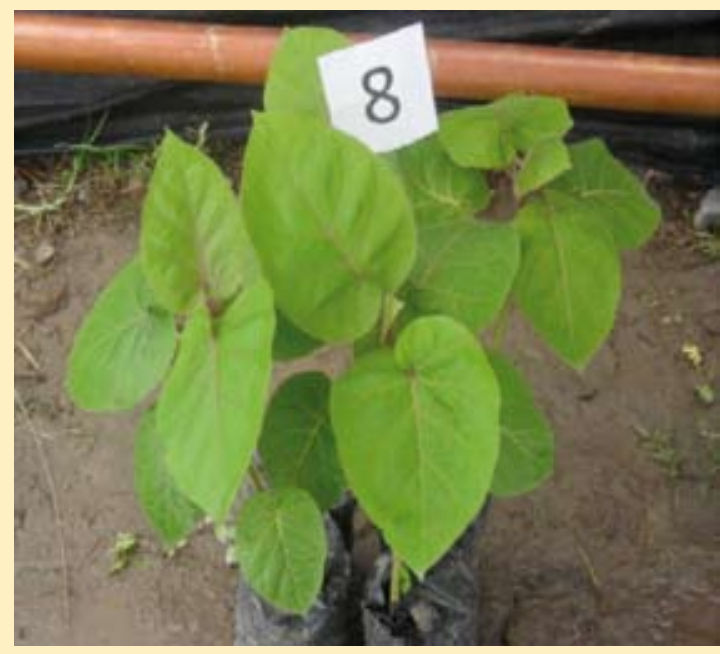

Figura 12. Fotos de plantas de Solanum betaceae con inóculos de esporas Nigrospora. Fuente: Ernesto Delgado Fernández

\subsection{Biomasa en fresco final}

La evaluación de la biomasa final, se valoró, mediante el peso de cada unidad experimental. El ANOVA realizado nos indica que existen diferencias significativas de acuerdo a los valores $(F=2,14 P=0,127)$, de acuerdo a este análisis, el tratamiento 2 Nigrospora Sp cepa A20 se presenta como el mejor, siendo estadísticamente altamente significativo, considerando la comparación con el testigo referencial Colletotrichum gloesporiodes. El tratamiento I Nigrospora. Sp: cepa AI 3 y el testigo absoluto tienen un comportamiento estadístico similar (Figura I3). 


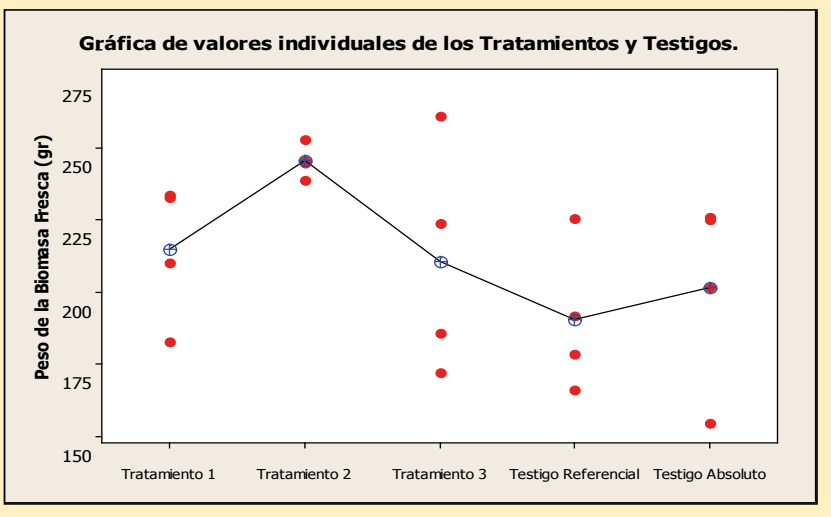

Figura 13. Biomasa Fresca, Medias del peso de la biomasa (gr) por cada tratamiento

\subsection{Altura Final}

Este parámetro se evalúa, midiendo desde el cuello hasta el ápice de todas las plantas, el ANOVA ( $F=$ 2,47 $\mathrm{P}=0,090$ ) nos indica que existen diferencias significativas, siendo el mejor tratamiento el número 2 que corresponde a Nigrospora sp: cepa A20 el testigo referencial difiere significativamente del resto de tratamientos. El tratamiento I, 2 y testigo absoluto, no presentan diferencias significativas (Figura 14).

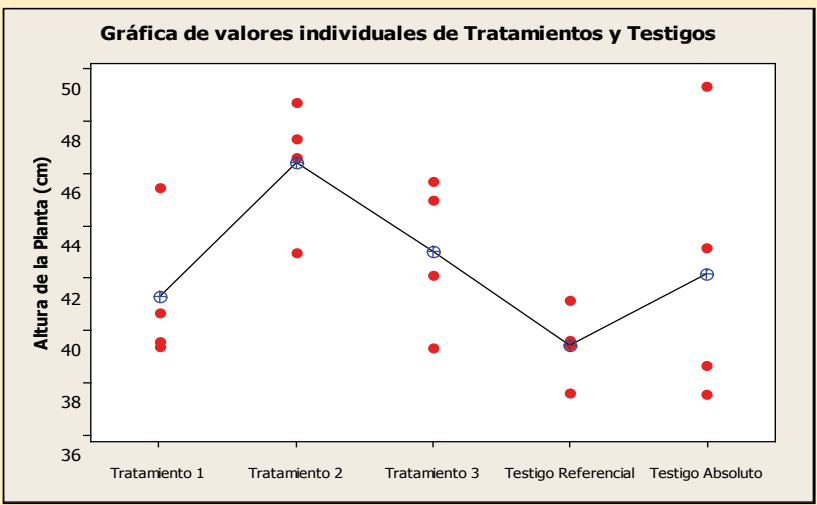

Figura 14. Medias de la altura de las Plantas $(\mathrm{cm})$ por cada Tratamiento

\section{Conclusiones y recomendaciones.}

Los tratamientos con agentes de biocontrol (hongos endófitos del género Nigrospora Sp.) penetraron en las plantas y se comportaron como agentes antagonistas de Colletotrichum. Las diferencias estadísticas resultan de la comparación con el testigo referencial (TR) (Colletotrichum) y el tratamiento 2 Nigrospora sp: cepa A20.
Los tratamientos I (Suspensión de esporas de Nigrospora. Sp: cepa Al3) y 3 (Suspensión de esporas micelio estéril, cepa $\mathrm{HXI}$ ) presentaron un comportamiento estadístico similar mientras que las plantas que corresponden al tratamiento 2 Nigrospora sp: cepa A20 presentan mayor vigor y desarrollo lo que corrobora el análisis estadístico realizado.

El efecto de los agentes biológicos utilizados como antagonistas (hongos endófitos) en el control biológico, de Colletotrichum participan en la promoción del crecimiento de las plantas y el uso de hongos endófitos antagonistas del género Nigrospora Sp., no afecta el desarrollo normal de la planta, en las condiciones que se llevó a cabo la investigación (temperatura y humedad relativa). Además que la infección por Colletotrichum gloesporoides influye en el metabolismo vegetal y producción de biomasa, según se evidencia en el análisis estadístico de biomasa.

Estos resultados evidencian la disminución y control de los daños causados por C. Gloesporoides en Solanum betaceum, en este sentido se observa que Nigrospora. Sp A20 se presenta como el mejor tratamiento, disminuyendo significativamente los síntomas de la Antracnosis. Podemos afirmar que dicha cepa no interfiere negativamente en la fisiología del cultivo, y posiblemente promueva un mejor desarrollo de la planta, ya que las plantas pertenecientes a este tratamiento se observaron con mayor vigor.

De acuerdo a la definición de endofitismo "microorganismos (hongos) que viven asintomáticamente en la planta, la mayor parte de su ciclo vital, tienen esporulación limitada en breves periodos y en algunos casos pueden estimular el desarrollo $y$ la "fuerza competitiva del huésped" podríamos concluir que es posible un beneficio indirecto de la planta huésped. Como resultado de esta investigación se considera, los tratamientos 1 y 2 a (Nigrospora. Sp A20) y (Nigrospora. Sp A/3) para posteriores estudios en campo abierto bajo condiciones ambientales normales, debido a su potencial antagónico frente a Colletotrichum.

Asimismo se recomienda probar inóculos combinados de hongos endófitos antagonistas. Así como el uso de pruebas moleculares y ADN ribosomal y el uso de marcadores ITS con el fin de verificar la presencia 
de los endófitos inoculados y/o del agente patógeno de la planta. Es recomendable también evaluar el potencial antagónico de los hongos endófitos, a campo abierto, $y$ en condiciones ambientales diversas y medir el uso de macro y micro nutrientes en la potenciación de la efectividad del biocontrol.

\section{Referencias}

Aponte,A., Debrot E., Arnal E., Solórzano R., y Ramos F. 2006. Diagnóstico de las enfermedades del Tomate de Árbol en los estados de Aragua y Miranda, Venezuela. Revista Ceniap Hoy. $N^{\circ}$ 9.Venezuela.

Barnett, H. y Hunter B. 1998. Illustrated Genera of Imperfect Fungi. Fourth Edition. Minnesota; Burgess Publishing Company. USA. 220p.

Bayley, A. y J. Jeger. 1992 Colletotrichum Biology, pathology and control. British Society for plant pathology C.A. B international 388p.

Botero. M. 1999. Estudios biológicos y epidemiológicos de la antracnosis (Colletotrichum gloeosporioides Penz) en tomate de árbol y desarrollo de alternativas para su manejo integrado en Colombia. Corporación Colombiana de Investigación Agropecuaria. 65p.

Cañedo, V. y Ames T. 2004. Manual de laboratorio para el manejo de hongos entomopatógenos. Centro Internacional de la Papa. Lima. Perú.

Ceron, L., Higuera B., Sánchez J., Bustamante S. y Buitrago G. 2006. Crecimiento y desarrollo de Colletotrichum gloeosporioides f. Alatae durante su cultivo en medios líquidos. Acta Biológica Colombiana,Vol. I I No. I.Universidad Nacional de Colombia, Bogotá, Colombia.

Chávez Méndez, NP. 2007. Utilización de bacterias y hongos endófitos para el control biológico del nematodo barrenador Radopholus similis (Cobb) Universidad de Chile
Delgado E. 2009 Actividad biológica de hongos endófitos presentes en dos plantas medicinales chuquiragua (Chuquiragua Jussiui J F Gmel) y Ñachag (Bidens andícola Kunth) La Granja 9(2). Ediciones ABYA-YALA Universidad Politécnica Salesiana

Finch, H.y Finch A. 1997. Los hongos comunes que atacan a los cultivos en América Latina. Segunda Edición. Editorial Trillas. México D.F. 189p.

Gams, W., Hoekstra E.S., Aptroot A. 1998. CBS Course of Mycology. Fourth Edition. Centraalbureau voor Schimmelcultures. Wageningen, the Netherlands. I55p.

Hawkworth D.L,. Kirk P.M., Sutton B.C. y Pegler D.N. 1995 Dictionary of the Fungi. Eighth Edition. International Mycological Institute. U.K. 6I6pg

Mendoza, J.2003. Control biológico del salivazo de la caña de azúcar (Mahanarva andigena). Memorias del Primer Seminario de Control Biológico. Universidad de Cuenca. Ecuador. 106 p.

Ramirez, J., Delgado E. y Tosi S. 2006 Actividad antagónica de hongos endófitos de plantas medicinales del Ecuador sobre bacterias patógenas. Boletín micológico Universidad de Valparaíso - Chile

Saldarriaga, A, Bernal J. y Tamayo P. 1997. Enfermedades del cultivo del tomate de árbol en Antioquia: Guía de reconocimiento y control. En: Boletín Técnico, Corporación Colombiana de Investigación Agropecuaria (Corpoica); C.I. La Selva, Rionegro (Antioquia). p. 8-I0. 